\title{
Molecular Investigations on tRNAs Involved in Human Mitochondrial Disorders
}

\section{Catherine Florentz}

Received November 26, 2001

\begin{abstract}
Over the last decade, human neurodegenerative disorders which correlate with point mutations in mitochondrial tRNA genes became more and more numerous. Both the number of mutations (more than 70) and the variety of phenotypes (cardiopathies, myopathies, encephalopathies as well as diabetes, deafness or others) render the understanding of the genotype/phenotype relationships very complex. Here we first summarize the efforts undertaken to decipher the initial impact of various mutations on the structure/function relationships of tRNAs. This includes several lines of research, namely (i) investigation of human mitochrondrial tRNA structures, (ii) comparison of disease-related and polymorphic mutations at a theoretical level, and (iii) experimental investigations of affected tRNAs in the frame of mitochondrial protein synthesis. A new approach aimed at searching for longrange effects of mitochondrial tRNA mutations on a broader global mitochondrial level will also be presented. Initial results obtained by comparative mitochondrial proteomics turn out to be very promising for deciphering unexpected molecular partners involved in the pathological status of the mitochondria.
\end{abstract}

KEY WORDS: tRNA structure; aminoacylation; translation; in vitro transcription; proteomics

\section{INTRODUCTION}

Transfer RNAs (tRNAs) are small ribonucleic acids (62 to 95 nucleotides) present in all organisms to ensure ribosomo-dependent protein biosynthesis (Söll and RajBhandary, 1995). Main aspects of their contribution to this process are to become charged with a specific amino acid by their cognate aminoacyl-tRNA synthetases (aaRSs), bind to messenger RNA, and transfer the amino acid to the nascent peptide chain at the level of the ribosome. More than 4300 sequences are referenced in a specific database, including prokaryotic, eukaryotic-cytosolic, -chloroplastic, and -mitochondrial (mt) molecules (Sprinzl et al., 1998). A large proportion of these tRNAs, the so called "canonical tRNAs," i.e., bacterial or eukaryotic cytosolic tRNAs, have been studied in much detail and their knowledge is of text-book value. Structural aspects (cloverleaf secondary structure, conserved sets of primary elements, tertiary interactions, L-shaped three-dimensional structures, ...)

UPR 9002 du CNRS, Département Mécanismes et Macromolécules de la Synthèse Protéique et Cristallogenèse, Institut de Biologie Moléculaire et Cellulaire du CNRS, 15, rue René Descartes, 67084 Strasbourg Cedex, France. Tel: 333884170 59; Fax: 333886022 18; E-mail: C.Florentz@ibmc.u-strasbg.fr

81 
as well as functional aspects (recognition by proteic partners such as aaRS, translation initiation or elongation factors, ribosomal protein, ...) are becoming deciphered. Best known are the aminoacylation "identity sets," elements allowing specific recognition by the cognate aaRS and hindering recognition by the other synthetases (Giegé et al., 1993; Giegé et al., 1998).

As opposed to canonical tRNAs, mt tRNAs form a structurally heterogeneous family, with more or less drastic divergences from the textbook situation discussed above, and are thus often considered as "bizarre" tRNAs (Dirheimer et al., 1995; Martin, 1995; Wolstenholme et al., 1987). Although a large number of mt tRNA sequences are presently known (about 2000) in the reference database (Sprinzl et al., 1998), both detailed structural and functional knowledge on this class of tRNAs remains far behind our understanding of canonical tRNAs. This is a drawback, especially in the case of human mt tRNAs, for which more and more correlation between point mutations in the corresponding genes and a variety of maternally inherited neuromuscular and neurodegenerative disorders have been established over the last 12 years (DiMauro and Andreu, 2000; DiMauro and Moraes, 1993; Larsson and Clayton, 1995; Schon, 2000; Schon et al., 1997; Wallace, 1999). These disorders include fatal cardiopathies, encephalopathies, myopathies, as well as deafness or diabetes and others. Since 1990, when the first correlation between the MELAS syndrome (mitochondrial encephalopathy with lactic acidosis and stroke-like episodes) and mutation A3243G in the tRNA ${ }^{\text {Leu(UUR) }}$ gene was described (Goto et al., 1990), more than 70 different mutations can now be referred to (Kogelnik et al., 1998). Understanding the relationship(s) between the different $\mathrm{mt}$ genotypes and the different phenotypes remains of major importance. The situation is complex since a given mutation can be associated with several types of pathologies, and, on the other hand, a given pathology can be linked to different mutations. Moreover, different degrees of severity in phenotypes can be observed for the same mutation. Whereas some of these findings can be explained by mitochondria-specific features such as mitotic segregation and threshold effect in heteroplasmy (i.e., the presence of both wild-type and mutated versions of $\mathrm{mt}$ genomes in a single cell), others remain unexplained.

In what follows, several directions of research developed to aid our understanding of the effects of point mutations in mt tRNA genes at a molecular level, will be presented and prominent results obtained in the field will be summarized. These include (i) structural understanding of mammalian $m t \mathrm{tRNAs}$ and theoretical analyses of mutations observed, (ii) defects of mutated tRNAs at the level of mt translation, and (iii) long-range cellular effects of tRNA mutations by comparative mt proteomics. Before discussing these research lines, the status of $\mathrm{mt} t \mathrm{RNAs}$ in a human cell will be recalled and the variety of mutations affecting these tRNAs in pathologies will be presented.

\section{HUMAN MITOCHRONDRIAL tRNAs AND THE NUCLEAR GENOME}

Human mitochondria possess a small circular DNA (16659 base-pairs) which encodes 13 protein genes (Anderson et al., 1981; Montoya et al., 1981). These proteins are subunits of 4 of the 5 transmembranar respiratory chain complexes involved 
in the major energy (ATP) synthesis pathway of the cell. They consist of 7 subunits of NADH-ubiquinone oxidoreductase (complex I), the cytochrome b apoprotein of ubiquinone-cytochrome c oxidoreductase (complex III), 3 subunits of cytochrome c oxidase (complex IV) and 2 subunits of ATP synthase (complex V). These mitochondrion-encoded subunits of the respiratory chain complexes are complemented by about 70 subunits encoded by the nuclear genome and imported from the cytosol (Fig. 1). For translation of the $13 \mathrm{mt}$ genes, the $\mathrm{mt}$ genome encodes also 2 ribosomal RNA genes and 22 tRNA genes, one tRNA specific for each of 18 amino acids, two specific for leucine (tRNA ${ }^{\text {Leu(UUR) }}$, tRNA ${ }^{\text {Leu(CUN) }}$ ) and two specific for serine $\left(\right.$ tRNA $^{\text {Ser(AGY) }}$, tRNA $^{\text {Ser(UCN) }}$ ) (the triplet indicated in brackets refers to the sequence of the codons complementary to the tRNA anticodon, with R for purine, $\mathrm{Y}$ for pyrimidine, and $\mathrm{N}$ for any of the 4 bases). This set of 22 is the minimal combination necessary to read all codons present in the 13 protein genes. Thus, the importance of each of these individual tRNAs in mt protein synthesis is well understandable.

The numerous functions of mitochondria (fatty acid degradation, Krebs cycle, urea cycle, heme biosynthesis, ubiquinol biosynthesis, etc., reviewed by Scheffler, 1999) can only be fulfilled thanks to the import of several hundred proteins from cytosol. These proteins include those required for the maintenance and expression of the mt genome (replication, transcription, translation), those involved in the many metabolic pathways, and those transmembranar proteins involved in the import mechanisms themselves. Estimates place the number of these proteins between 600 and 2000. The mechanisms of cross-talk between the $\mathrm{mt}$ and nuclear genomes to regulate the adequate number of $\mathrm{mt}$ proteins remain mostly unknown.

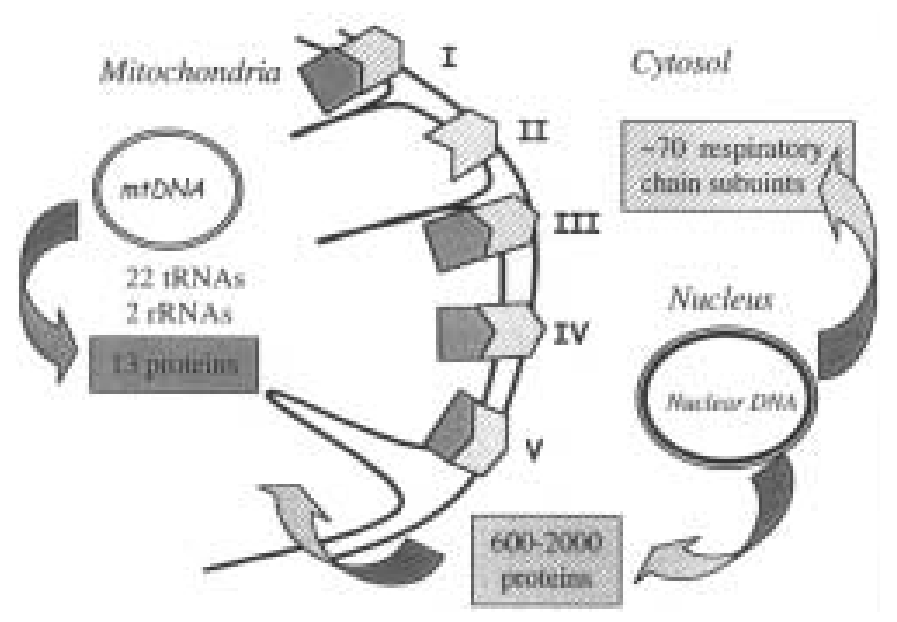

Fig. 1. Mitochondrial tRNAs in the cell. The human $m t$ genome encodes $22 \mathrm{tRNAs}$ involved in the $\mathrm{mt}$ translation process, and thus in the synthesis of 13 proteins, all subunits of respiratory chain complexes (schematized by gray arrowheads). These proteins are complemented by $\sim 70$ nuclear-encoded subunits, imported from the cytosol (dashed arrowheads) to allow formation of active trans-membrane respiratory chain complexes. These complexes are involved in electron transfer and ATP synthesis. Mitochondria contain several hundreds of additional proteins synthesized in the cytosol and imported. These include, in particular, proteins involved in mt translation. 
In conclusion, it appears that mt tRNAs are of critical importance at different levels. They are indispensable individual actors in the $\mathrm{mt}$ translation process. Moreover, since the synthesized $\mathrm{mt}$ respiratory chain subunits are directly linked both physically and functionally with nuclear encoded partners, and since the corresponding enzymatic complexes are located on the border line between the mt matrix and the cellular cytosol, mt tRNAs may swell influence long-range cellular processes far beyond mitochondria.

\section{PATHOGENIC MUTATIONS IN MITOCHONDRIAL tRNA GENES}

Mt DNA undergoes mutation at a much higher rate than nuclear genomes (e.g., Allen and Raven, 1996; Pesole et al., 1999). While some of the mutations are neutral (polymorphic mutations) and contribute to the diversity of human populations (e.g., Ingman et al., 2000; Krings et al., 1997), many are correlated to disease (e.g., DiMauro and Moraes, 1993; Larsson and Clayton, 1995; Schon et al., 1997). Table 1 recalls the subset of mutations described in tRNA genes linked to disorders. So far, 20 out of $22 \mathrm{mt}$ tRNA genes have been shown to be affected by mutations, and it is likely that mutations within the two remaining genes ( $\mathrm{tRNA}^{\mathrm{Arg}}$ and $\mathrm{tRNA}^{\mathrm{His}}$ ) will be described in the future. Whereas certain genes such as those for tRNA ${ }^{\text {Leu(UUR) }}$, $\mathrm{tRNA}^{\mathrm{Lys}}$, and $\mathrm{tRNA}^{\mathrm{Ile}}$ appear as hot spots for mutations, others are less often hit.

\section{INVESTIGATION OF MITOCHONDRIAL tRNAs AND MITOCHONDRIAL TRNA MUTATIONS ON A THEORETICAL LEVEL}

\section{Structural characteristics of mammalian mitochondrial tRNAs}

Structure is the basis for function. Understanding the structure of mt tRNAs will be the basis for understanding their function and will probably help us to understand the effects of point mutations at a molecular level. Already while sequencing the human $\mathrm{mt}$ genome in the early $80 \mathrm{~s}$, Anderson and colleagues noticed some unusual features of the tRNA genes, including the absence of a complete structural domain for one of the serine-specific tRNAs and the absence of some typically conserved sequences for other tRNAs (Anderson et al., 1981). For example, a UUC sequence classically converted into the TYC sequence by post-transcriptional modification, was absent in most genes. Subsequently, the structures of three mammalian $m t$ tRNAs have been experimentally investigated, namely tRNAs ${ }^{\text {Ser(AGY) }}{ }^{\text {(de Bruijn }}$ and Klug, 1983; Hayashi et al., 1997; Ueda et al., 1983), tRNA ${ }^{\text {Phe }}$ (Wakita et al., 1994), and tRNA ${ }^{\mathrm{Ser}(\mathrm{UCN})}$ (Hayashi et al., 1998; Watanabe et al., 1994), confirming unusual folding rules for these RNAs. Complementary knowledge on structural characteristics of each of the other human $\mathrm{mt}$ tRNAs came from theoretical analysis based on the compilation of the 22 tRNA genes in 31 fully sequenced mammalian $\mathrm{mt}$ genomes (Helm et al., 2000). Vertical alignments allowed us to deduce that, with the exception of the tRNA ${ }^{\text {Ser(AGY) }}$ group, all families of tRNAs do fold into cloverleaf structures. However, these cloverleaves differ from the canonical ones by large size variations, especially in the T-loops (Fig. 2). This is a striking feature since 
Table 1. Disease-related mutations in human mitochondrial tRNA genes

\begin{tabular}{|c|c|c|c|c|}
\hline \multirow{2}{*}{$\begin{array}{l}\text { Amino acid } \\
\text { specificity }\end{array}$} & \multirow[b]{2}{*}{ Gene mutation } & \multicolumn{2}{|c|}{ tRNA mutation } & \multirow[b]{2}{*}{ Related pathologies } \\
\hline & & domain & position & \\
\hline Ala & A5628G & AC stem & $31-39$ & CPEO \\
\hline \multicolumn{5}{|l|}{ Arg } \\
\hline \multirow[t]{3}{*}{ Asn } & A5692G & AC loop & 38 & CPEO \\
\hline & $\mathrm{C} 5698 \mathrm{~T}$ & AC loop & 32 & PEO \\
\hline & C5703T & AC stem & $27-43$ & CPEO, MM \\
\hline Asp & A7543G & AC stem & $29-41$ & MS \\
\hline Cys & A5814G & D-stem & $13-22$ & EM, MELAS, PEO \\
\hline \multirow[t]{3}{*}{ Gln } & $\mathrm{C} 4332 \mathrm{~T}$ & acc. stem & $3-70$ & EM, D \\
\hline & A4336G & acc. stem & $7-66$ & ADPD \\
\hline & instT4370 & AC loop & after 31 & $\mathrm{MM}, \mathrm{CD}$ \\
\hline Glu & A14709G & AC loop & 37 & MM, EM, D \\
\hline \multirow[t]{4}{*}{ Gly } & T9997C & acc. stem & $7-66$ & MHCM \\
\hline & A10006G & D-loop & 18 & CIPO \\
\hline & $\mathrm{T} 10010 \mathrm{C}$ & D-stem & $12-23$ & EM \\
\hline & A10044G & T-loop & 59 & EM \\
\hline \multicolumn{5}{|l|}{ His } \\
\hline \multirow{7}{*}{ Ile } & A $4269 \mathrm{G}$ & acc. stem & $7-66$ & FICP, EM \\
\hline & $\mathrm{T} 4274 \mathrm{C}$ & D-stem & $13-22$ & CPEO \\
\hline & $\mathrm{T} 4285 \mathrm{C}$ & AC stem & $27-43$ & PEO \\
\hline & G4298A & $\mathrm{AC}$ stem & $30-40$ & CPEO \\
\hline & G4309A & T-stem & $51-63$ & CPEO \\
\hline & $\mathrm{A} 4317 \mathrm{G}$ & T-loop & 59 & FICP \\
\hline & $\mathrm{C} 4320 \mathrm{~T}$ & T-stem & $52-62$ & ECM \\
\hline \multirow[t]{4}{*}{ Leu(CUN) } & $\mathrm{T} 12297 \mathrm{C}$ & Ac loop & 33 & DCM \\
\hline & G12301A & AC loop & 37 & AISA \\
\hline & G12415A & T-stem & $52-62$ & CPEO \\
\hline & A12320G & T-loop & 57 & $\mathrm{MM}$ \\
\hline \multirow[t]{20}{*}{ Leu(UUR) } & A $3243 \mathrm{G}$ & D-loop & 14 & MELAS, DMDF \\
\hline & A $3243 \mathrm{~T}$ & D-loop & 14 & PEM, MM \\
\hline & G3249A & D-loop & 19 & $\mathrm{KS}$ \\
\hline & $\mathrm{T} 3250 \mathrm{C}$ & D-loop & 20 & MM \\
\hline & A $3251 G$ & D-loop & 20: 01 & MM \\
\hline & A3252G & D-loop & 21 & MELAS \\
\hline & $\mathrm{C} 3254 \mathrm{G}$ & D-stem & $12-23$ & $\mathrm{MM}$ \\
\hline & $\mathrm{C} 3256 \mathrm{~T}$ & D-stem & $10-25$ & MERRF-like, MELAS \\
\hline & $\mathrm{T} 3258 \mathrm{C}$ & AC stem & $27-43$ & LA, E1 \\
\hline & A $3260 \mathrm{G}$ & $\mathrm{AC}$ stem & $29-41$ & MMC \\
\hline & T3264C & AC loop & 33 & $\mathrm{DM}$ \\
\hline & $\mathrm{T} 3271 \mathrm{C}$ & AC stem & $30-40$ & MELAS, DM \\
\hline & delT3272 & AC stem & $29-41$ & PEM \\
\hline & T3273C & AC stem & $28-42$ & $\mathrm{O}, \mathrm{EI}$ \\
\hline & $\mathrm{C} 3275 \mathrm{~A}$ & var. region & 44 & LHON \\
\hline & A $3280 \mathrm{G}$ & T-stem & $49-65$ & MM \\
\hline & A $3288 \mathrm{G}$ & T-loop & 57 & MM \\
\hline & T3291C & T-loop & 60 & MELAS \\
\hline & A $3302 \mathrm{G}$ & acc. stem & $2-71$ & $\mathrm{MM}$ \\
\hline & $\mathrm{C} 3303 \mathrm{~T}$ & acc. stem & $1-72$ & MMC \\
\hline \multirow[t]{3}{*}{ Lys } & A8296G & acc. stem & $2-71$ & DMDF, MERRF \\
\hline & G8313A & D-stem & $12-24$ & MNGIE \\
\hline & $\mathrm{T} 8316 \mathrm{C}$ & AC stem & $27-43$ & MELAS \\
\hline
\end{tabular}


Table 1. Continued

\begin{tabular}{|c|c|c|c|c|}
\hline \multirow{2}{*}{$\begin{array}{l}\text { Amino acid } \\
\text { specificity }\end{array}$} & \multirow[b]{2}{*}{ Gene mutation } & \multicolumn{2}{|c|}{ tRNA mutation } & \multirow[b]{2}{*}{ Related pathologies } \\
\hline & & domain & position & \\
\hline & G8328A & AC stem & $31-39$ & EM \\
\hline & G8342A & T-stem & $53-61$ & PEO, MS \\
\hline & A8344G & T-loop & 55 & MERRF \\
\hline & $\mathrm{T} 8355 \mathrm{C}$ & T-stem & $50-64$ & $\mathrm{PEO}, \mathrm{SM}$ \\
\hline & $\mathrm{T} 8356 \mathrm{C}$ & T-stem & $49-65$ & MERRF \\
\hline & $\mathrm{T} 8362 \mathrm{G}$ & acc. stem & $2-71$ & $\mathrm{SM}$ \\
\hline & G8363A & acc. stem & $1-72$ & MICM, D, MERRF, LS \\
\hline \multirow[t]{2}{*}{ Met } & $\mathrm{T} 4409 \mathrm{C}$ & acc./D-stem & 8 & $\mathrm{MM}$ \\
\hline & G4450A & T-stem & $53-61$ & $\mathrm{MM}$ \\
\hline \multirow[t]{3}{*}{ Phe } & G583A & acc. stem & $7-66$ & MELAS \\
\hline & A606G & $\mathrm{AC}$ stem & $29-41$ & M \\
\hline & T618C & AC stem & $29-41$ & $\mathrm{MM}$ \\
\hline \multirow[t]{2}{*}{ Pro } & $\mathrm{T} 15965 \mathrm{C}$ & T-stem & $50-64$ & ADPD \\
\hline & G15990A & AC loop & 36 & MM, O \\
\hline \multirow[t]{2}{*}{$\operatorname{Ser}(\mathrm{AGY})$} & C12246A & T-loop & 55 & CIPO \\
\hline & $\mathrm{C} 12258 \mathrm{~A}$ & acc. stem & $7-66$ & DMDF \\
\hline \multirow[t]{4}{*}{$\operatorname{Ser}(\mathrm{UCN})$} & insG7472 & var. region & 46 & PEM \\
\hline & $\mathrm{C} 7497 \mathrm{~T}$ & D-stem & $13-22$ & MM, PEM, RRF, LA \\
\hline & A7511G & acc. stem & $4-69$ & DEAF, SNHL \\
\hline & A7512G & acc. stem & $3-70$ & PEM \\
\hline \multirow[t]{4}{*}{ Thr } & G15915A & $\mathrm{AC}$ stem & $30-40$ & MM \\
\hline & A15923G & AC loop & 38 & LIMM \\
\hline & delT15940 & T-loop & 60 & MM \\
\hline & G15950A & acc. stem & $3-70$ & ADPD \\
\hline \multirow[t]{4}{*}{ Trp } & G5521A & D-stem & $10-25$ & $\mathrm{MM}$ \\
\hline & insT5537 & AC stem & after 27 & MILS \\
\hline & G5540A & AC stem & $30-40$ & PEM, CD \\
\hline & G5549A & AC stem & $31-39$ & DEMCHO, D, A \\
\hline Tyr & A5874G & D-stem & $13-22$ & EI, LW, CD \\
\hline \multirow[t]{3}{*}{ Val } & G1606A & acc. stem & $5-68$ & AMDF \\
\hline & G1642A & $\mathrm{AC}$ stem & $27-43$ & MELAS \\
\hline & G1644T & var. region & 45 & LS \\
\hline
\end{tabular}

Data have mainly been retrieved from updated database http://infinity.gen./emory.edu/mitomap.html. tRNA genes are listed by amino acids specificity in alphabetical order. "Gene mutation" refers to the nucleotide substition and position of the mutation in the human mt genome. "tRNA mutation" refers to the location of the mutation in the gene product, the acutual tRNA. Structural domains affected by the mutations refer to loops and stems according to Fig. 2, with AC for anticodon, acc. for acceptor, var. for variable. Nucleotide numbering is according to classical tRNA numbering (Sprinzl et al., 1998). Numbers in bold correspond to the affected positions. Pathologies are abbreviated as follows: A-Ataxia; ADPD-Alzeimer's Disease and Parkinsons's Disease; AISA-Acquired Idiopathic Sideroblastic Anemia; AMDF-Ataxia, Mental deterioration, DeaFness; CD-Cox Deficiency; CIPO-Chronic Intestinal PseudoObstruction with myopathy; CPEO-Chronic Progressive External Ophtalmoplegia; D-Diabete; DCM-Dilated CardioMyopathy; DEAF-Maternally inherited DEAFness or aminoglycoside-induced DEAFness; DEMCHO-DEMentia, CHOrea; DM-Diabetes Mellitus; DMDF-Diabetes Mellitus, DeaFness; ECM-EncephaloCardioMyopathy; EI-Exercise Intolerance; EM-EncephaloMyopathy; FICP-Fatal Infantile Cardiomyopathy Plus a Melas-associated cardiomyopathy; HCM-Hypertrophic CardioMyopathy; KS-Kearns-Sayre syndrome; LA-Lactic Acidose, LHON-Leber Hereditary Optic Neuropathy; LIMM-Lethal Infantile Mitochondrial Myopathy; LS-Leigh Syndrome; LW-Limb Weakness; M-Myoglobinuria; MELAS-Mitochondrial Encephalomyopathy, Lactis Acidose, Stroke-like episodes; MERRF-Myoclonic Epilepsy and Ragged Red muscle Fibers; MHCM-Maternally inherited Hypertrophic CardioMyopathy; MICMMaternal; Inherited CardioMyopathy; MILS-Maternal Inherited Leigh Syndrome; MM-Mitochondrial Myopathy; MMC-Maternal Myopathy and Cardiomyopathy; MNGIE-Mitochondrial NeuroGastroIntestinal Encephalomyopathy; MS-Myoclonic Seizures; O-Ophtalmoplegia; PEM-Progressive EncephaloMyopathy; PEOProgressive External Ophtalmoplegia; SM-Skeletal Myopathy; SNHL-SensoriNeural Hearing Loss. 
T-loops are strictly conserved in size and strongly conserved in sequence in canonical tRNAs. A second striking feature is that all $\mathrm{mt}$ tRNAs have a "variable region" which is restricted to 3 to 5 nucleotides, whereas in tRNAs of other origins, it may have up to 23 nucleotides (Sprinzl et al., 1998).

Once secondary structural elements were aligned, it became possible to compare primary sequences and establish consensus sequences for each of the 22 tRNA families. Interestingly, the degree of conservation of nucleotides within a given family ranges from "very high conservation" (e.g., tRNA ${ }^{\mathrm{Leu}(\mathrm{UUR})}, \mathrm{tRNA}^{\mathrm{Met}}, \mathrm{tRNA}^{\mathrm{Ile}}$ ) to "very poor conservation" (e.g., tRNA ${ }^{\text {Lys }}$, tRNA ${ }^{\text {Asp }}$ ) (see examples in Fig. 2). Noteworthy is the rarity of classically conserved nucleotides. Thus, for example, the G18G19 doublet of the D loop is only present in 4 families out of 22, the UUC sequence in the T-loop is rarely present, individual nucleotides such as A14, R15, A21, and Y48 which are conserved in canonical cytosolic and bacterial tRNAs, are also often replaced by non-conserved nucleotides. Since these nucleotides are classically involved in tertiary interactions and thus in 3D folding, it can be concluded that folding rules for mammalian mt tRNAs, and thus for human mt tRNAs, are probably different than for classical tRNAs, and remain to be discovered.

Establishment of the consensus secondary structures of the 22 mammalian $\mathrm{mt}$ tRNAs provides a basis for experimentation and increases the fundamental knowledge on structure/function relationships of these molecules, with the goal of deciphering the impact of mutations on these relationships.

\section{Search for features common to the set of pathology-related mutations}

Many polymorphic mutations in tRNA genes have been reported (e.g., Ingman et al., 2000). Basic features of these mutations as well as of disease-related mutations have been compiled and compared, with the aim of highlighting significant differences (Florentz and Sissler, 2001). Since conserved nucleotides are expected to be of major importance in either structural or functional properties of a molecule, individual pathogenic and polymorphic mutations have been overimposed on the cognate consensus sequences for mammalian mt tRNAs (Helm et al., 2000). As expected, most pathogenic mutations affect highly conserved nucleotides whereas most polymorphic mutations affect rather non-conserved nucleotides. However, exceptions exist, i.e., some pathogenic mutations do affect non-conserved positions and some polymorphic mutations do affect conserved mutations, suggesting that the degree of conservation of a nucleotide cannot be taken as a guarantee that the mutation will be pathology-related.

Further comparison of pathogenic and polymorphic mutations concerned their chemical type (transition, transversion), their distribution on the secondary structural domains (location in single-stranded domains), and their structural effect in stems (type of base-pair change introduced). These simple criteria were not sufficient to establish striking differences between both families of mutations. All mutations are distributed rather uniformly throughout the tRNAs in both stems and loops, they consist largely of transitions rather than transversions, and in stems, convert Watson-Crick pairs into structurally mild CA or GU mismatches. This analysis 
showed that case by case molecular investigation remains necessary to assess the growing family of pathogenic mutations in $\mathrm{mt}$ tRNAs.

\section{SEARCH FOR EFFECTS OF MUTATIONS ON VARIOUS ASPECTS OF MITOCHONDRIAL TRANSLATION}

Considering the central role of tRNA in protein biosynthesis, obvious targets for the investigation of mutational effects are structural and functional aspects of the tRNA in the frame of mt translation. Global effects on translation were indeed reported in a number of cases (e.g., Enriques et al., 1995; Hao and Moraes, 1996; King et al., 1992; Seibel et al., 1991). Several approaches have been developed to attempt to sort out at which precise level of tRNA biogenesis and/or function the mutations are harmful.

In vitro analyses were based on tRNA preparation by cloning the corresponding synthetic genes downstream from an efficient RNA polymerase promoter and in vitro transcription. Such an approach, giving access to any tRNA sequence in sufficient amounts, had been successfully used with a large number of canonical tRNAs and led in particular to finding aminoacylation identity elements (reviewed in Giegé et al., 1998). In vitro transcripts of human mt tRNAs lead to a number of surprises. In the case of $\mathrm{tRNA}^{\mathrm{Lys}}$, it became clear that the absence of post-transcriptional modifications was deleterious to a correct folding of the tRNA (Helm et al., 1998). Only once a single methyl group is introduced (position 1 of adenine 9), the RNA chain folds into a cloverleaf structure (Helm et al., 1999b). In parallel to structural weakness, transcripts also revealed non optimal in function. Thus, aminoacylation capacity of unmodified tRNA ${ }^{\mathrm{Lys}}$ transcripts remains very poor (Tolkunova et al., 2000). Further negative results on aminoacylation of in vitro transcribed mt tRNAs were observed for several aminoacylation systems (our unpublished results) and for tRNA $^{\text {Phe }}$ (Bullard et al., 1999). In the case of tRNA ${ }^{\text {Ile }}$, aminoacylation was possible but with an efficiency 48-fold decreased in comparison to aminoacylation of the corresponding full post-transcriptionally modified natural tRNA extracted from placental mitochondria (Degoul et al., 1998). These examples show that the in vitro approach of tRNA preparation has some unexpected limitations. They also contributed strongly to the conclusion that post-transcriptional modifications are much more important for human mt tRNAs than for canonical tRNAs. As a consequence, it was hypothesized that pathogenic mutations could interfere with the optimal maturation of the tRNAs, i.e., their post-transcriptional modification (see below).

Use of in vitro transcribed tRNAs could, however, be further exploited in the case of tRNA ${ }^{\text {Ile }}$ to study the effects of various mutations on the aminoacylation capacities of the tRNA (Degoul et al., 1998; Kelley et al., 2000; Kelley et al., 2001). As a main outcome, it was found that all mutations analyzed do affect aminoacylation by the cognate enzyme in a more or less dramatic way by perturbing the structure and/or stability of the substrate tRNAs. Moreover, some mutated tRNAs become inhibitors of the aminoacylation of wild-type tRNAs (Kelley et al., 2000). This discovery highlights a new possibility of interference of mutated and wild-type tRNAs in patient mitochondria. Indeed, in cases of mt heteroplasmy, it was proposed so far that below a given threshold of mutated mt DNA, wild-type verions of 
the specific tRNA would compensate for the defective mutated tRNA. The wildtype tRNA ${ }^{\text {Ile }}$ may well not be able to compensate for mutated versions, but on the contrary, become inhibited by the defective molecules (Kelley et al., 2000).

Analysis of natural wild-type and mutated tRNAs extracted from cybrid celllines confirmed to some extent the possibility that mutations linked to pathologies can interfere with post-transcriptional modifications. Experiments were performed to explore tRNA ${ }^{\text {Lys }}$ mutated at position 8344 (related to the MERRF syndrome, see Table 1) and tRNA ${ }^{\text {Leu(UUR) }}$ mutated at position 3243 (related to the MELAS syndrome). A detailed quantitative comparison of each post-transcriptional modification present in wild-type and mutated $\operatorname{tRNA}^{\mathrm{Lys}}$ (6 modifications) and in wild-type and mutated tRNA ${ }^{\text {Leu(UUR) }}$ (9 modifications) extracted from osteosarcoma cell lines, revealed that none of the modifications is affected by the mutation in $\mathrm{TRNA}^{\text {Lys }}$ and that one specific modification, $\mathrm{m} 2 \mathrm{G} 10$, is reduced by $50 \%$ in the mutated tRNA $^{\text {Leu(UUR) }}$ (Helm et al., 1999a). The level of post-transcriptional modifications at the anticodon wobble position (position 34), a position known as important for codon reading, was difficult to investigate but was evaluated as poorly modified in both wild-type and mutated tRNA ${ }^{\text {Lys }}$ and tRNA ${ }^{\text {Leu(UUR) }}$ (Helm et al., 1999a). A distinct technique of post-transcriptional modification analysis, applied to tRNAs extracted from HeLa cells derived cybrids, brought information only on these these positions (Yasukawa et al., 2000b; Yasukawa et al., 2000c). It was found that whereas wild-type tRNA ${ }^{\text {Lys }}$ and tRNA ${ }^{\text {Leu(UUR) }}$ are fully modified at position 34, mutated versions of the tRNAs are not at all. It has further been demonstrated that the mutated tRNAs are unable to bind to ribosomes (Yasukawa et al., 2001). Although the two approaches applied to investigate the post-transcriptional modifications in mutation-carrying tRNAs lead to variable results, they both argue in favor of a correlation between mutations and post-transcriptional modification levels.

Further analyses on natural human mt tRNAs (extracted from cybrids or from biopsies) confirm and extend the impact of mutations to tRNA structure and biogenesis as well as to function. Indeed, abnormal transcription, e.g., (Bindoff et al., 1993; King et al., 1992; Koga et al., 1995; Schon et al., 1992), incomplete maturation (Fierl et al., 1997; Levinger et al., 2001; Rossmanith and Karwan, 1998), incomplete post-transcriptional modification (see above), and lower stability (Chomyn et al., 2000; Enriquez et al., 1995; Hao and Moraes, 1997; Yasukawa et al., 2000a) were observed for mutated tRNAs as compared to corresponding wild-type reference. The aminoacylation capacity of mutated tRNAs was found to be less efficient and/ or less specific (Chomyn et al., 2000; Enriquez et al., 1995), or at opposite not affected (Börner et al., 2000; Yasukawa et al., 2001). In some cases, frameshifting (Masucci et al., 1995; Masucci and Schon, 1996) or slower polysome formation (Chomyn et al., 2000) has been observed.

In summary, our present knowledge on the molecular impact of point mutations on human mt tRNA structure and function in translation is still puzzling. Whereas so far only a subset of mutations has been experimentally analyzed, effects reported are rather variable. The variability concerns the type of effect as well as the quantitative aspects which are likely linked to the nuclear backgrounds of the investigated cells (different cybrid cell lines, biopsies). However, despite the apparently conflicting 
results, many efforts are being made to try to solve the controversies and understand the diversity in molecular effects of mt tRNA mutations (e.g., Jacobs and Holt, 2000). While, so far, all molecular investigations have focussed on possible effects of mutations on conventional aspects of tRNAs in the frame of mt translation, it is possible that "the primary effect of the mutation on tRNA function may yet prove to be something that nobody has yet thought of." Such as hypothesis, proposed by several authors during the last decade and recalled provocatively recently (Jacobs and Holt, 2000), is indeed a challenging complementary possibility.

In what follows, a new approach of comparative $\mathrm{mt}$ proteomics will be described. Instead of searching for unexplored primary effects of mutations, this technique will investigate long-range secondary effects at the level of mitochondria as a global entity, and on the mt/nuclear genomes relationship. Such a global approach should allow to highlight alternative pathways to $\mathrm{mt}$ translation, which may be affected by the tRNA mutations. First promising results are presented.

\section{SEARCH FOR LONG-RANGE EFFECTS OF tRNA MUTATIONS BY A COMPARATIVE PROTEOMIC APPROACH}

Biochemical analyses of disease-related mitochondria generally highlight decreased oxygen consumption, decreased activities of respiratory chain complexes, and as a consequence, decreased rates of ATIP synthesis. Additional biological changes have been observed including lactic acidosis (Wallace, 1999), increased oxidative stress (Wallace, 1999), altered membrane potential (Antonicka et al., 1999) and apoptotic events (Asoh et al., 1996; Mirabella et al., 2000). Thus, additional molecular consequences of the primary effects of mutations occur in mitochondria as well as at the more global cellular level. These considerations suggest that global approaches, in addition to direct investigations, are required to understand the molecular effects of individual mutations in mt tRNAs and the relationship of these effects with the phenotypic expression of the disease. To test such possibilities, we have initiated a comparative proteomic approach on mitochondria extracted from couples of cybrid cell-lines representative of two point mutations, namely the MERRF related mutation 8344 and the MELAS related mutation 3243 (Rabilloud et al., 2002).

Proteomics consist of a high resolution separation of thousands of proteins at once, by $2 \mathrm{D}$ separation on polyacrylamide gels, and amino acid analysis (e.g., Wilkins et al., 1997). In a first dimension, proteins are separated according to their isoelectric point ( $\mathrm{pI}$ ) and in second dimension, according to their mass. This technique pioneered 20 years ago by O'Farrell (O'Farrell, 1995), gained much focus recently thanks to technological improvements combined with sensitive mass-spectrometric measurements allowing the assignment of individual proteins. To search for long-range effects of point mutations in mt tRNAs on the total mt protein content, we have explored the feasibility of comparative proteomics. This is based on a precise quantitative comparison of 2D maps obtained from mitochondria prepared from related cybrid cell-lines, cells with a same nuclear background and different only at the level of the single point mutation in the gene of one given mt tRNA. 
The reference cell-line carries the wild-type tRNA gene, the other cell-line carries the mutated gene.

\section{Long-range effects of mitochondrial tRNA mutations on nuclear-encoded proteins}

2D separation of the human $\mathrm{mt}$ proteins has been pioneered by Rabilloud and colleagues (Rabilloud et al., 1998), with much emphasis on solubilizing the numerous membrane proteins. While this still remains a limitation, it is however possible to observe about 1000 well-separated protein spots on a single silver-stained gel. When applied to mitochondria extracted from couples of related cybrid representative of mutation A8344G linked to the MERRF syndrome, and of mutation A3243G related to the MELAS syndrome, about 1000 spots are also observed (Fig. 3). While most spots are of similar intensity, numerous differences between the patterns of wild-type and mutation-carrying mitochondria can be observed by simple visual inspection, demonstrating that both down- and up-regulations of several proteins take place in the affected mitochondria. As illustrated in Fig. 3 for a subset of proteins, two spots, $\mathrm{X}$ and $\mathrm{Y}$, undergo drastic down-regulation in mitochondria related both to the MERRF and to the MELAS syndrome. Assignment of the proteins present in these spots by a combination of mass spectrometric techniques (MALDI-TOF, MS-MS) and Edman degradation revealed two nuclear-encoded subunits of a respiratory chain complex, cytochrome c oxidase (COX subunits $\mathrm{Vb}$ and VIa). COX consists of 13 subunits, 3 mitochondrially encoded and 10 of nuclear origin (Scheffler, 1999). It is already well established that both MERRF mutation A8344G and MELAS mutation A3243G interfere with the synthesis of the mt subunits, COX-I, COX-II, and COX-III (e.g., Enriquez et al., 1995; Chomyn et al., 2000). The comparative proteomic approach reveals that at least two additional subunits are affected, although they are nuclear-encoded. This was an initial step confirming that there are long-range consequences of a mutation in a mt tRNA gene. Interestingly, the same two proteins are affected for the two tRNA mutations considered, highlighting the possibility of common molecular mechanisms in the phenotypic expression of both MELAS and MERRF syndromes.

The next step in the proteomic approach will be complete computer assisted quantitative comparison of $2 \mathrm{D}$ patterns, and thus detection of those individual proteins which undergo the most important down- or up-regulation. It will then be of highest interest to assign these proteins by mass spectrometry and verify if these proteins further belong to nuclear-encoded respiratory chain subunits of if they correspond to enzymes of metabolic pathways, to proteins involved in $\mathrm{mt}$ translation or to other proteins. Whatever the surprise will be, knowledge of these proteins will enrich our global view of the major events taking place in the mutation-carrying mitochondria. These events may reveal further negative consequences of the mutations and/or compensatory events established by the cell to somehow overcome the primary effects of the mutation.

Long-range effects between $\mathrm{mt}$ and nuclear genomes have already been demonstrated, as for example, after complete blocking of $\mathrm{mt}$ genome expression ( $\rho^{\circ}$ cells), or after complete inhibition of mt translation by antibiotics (Nijtmans et al., 1995). However, the approaches used so far to detect affected proteins (specific antibodies) 

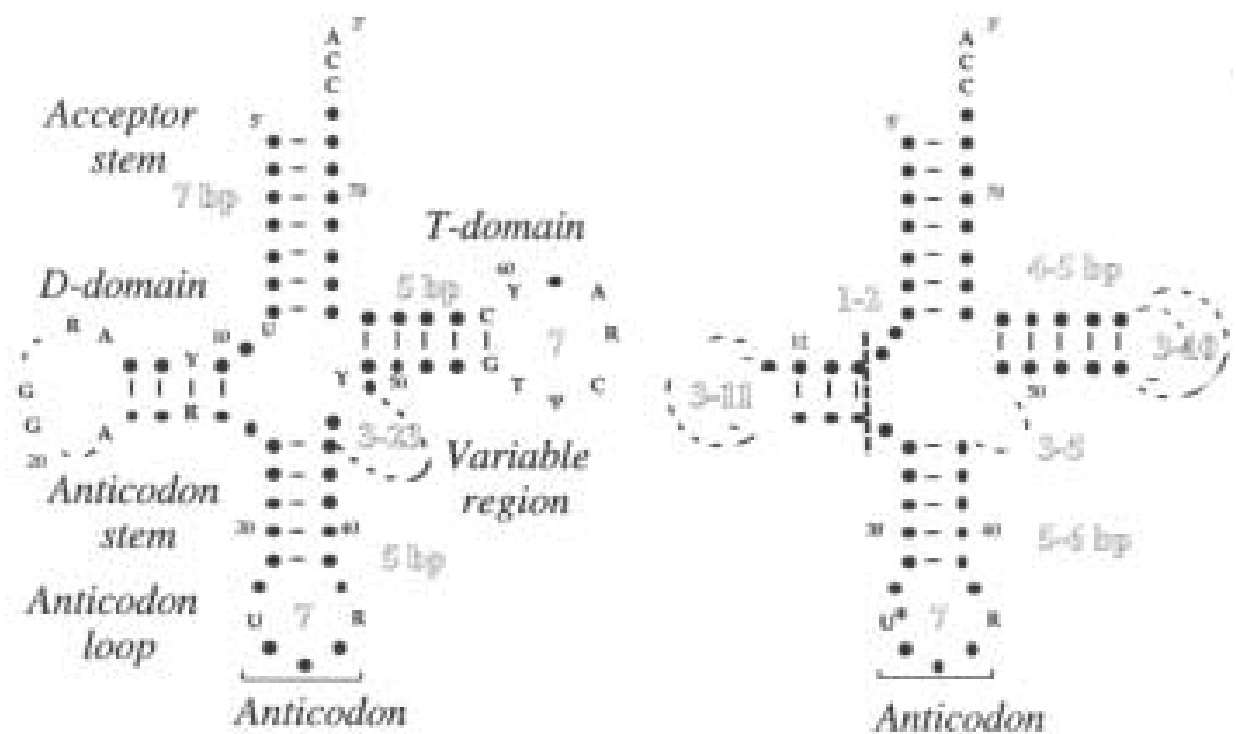

Anticodon

B.

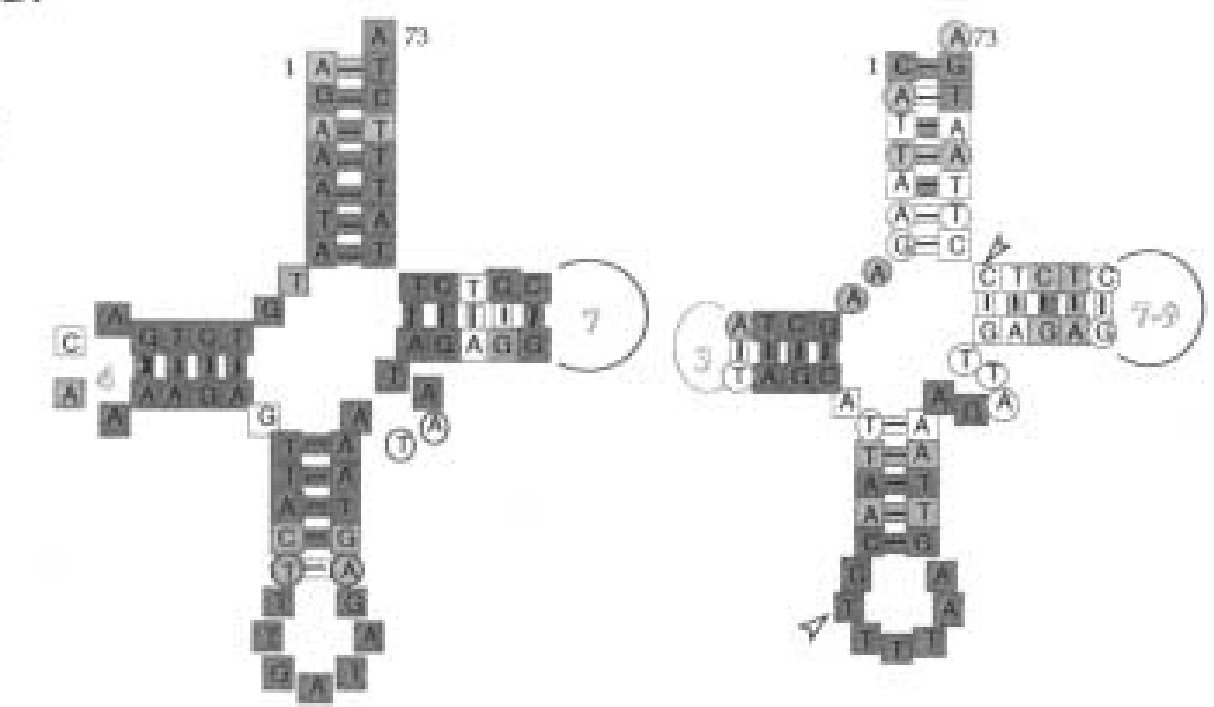

Fig. 2. Structural features of mammalian mitochondrial tRNAs. (A) Typical secondary structure of mammalian mt tRNAs (right) as compared to "classical" tRNA (left). Especially striking are the size variability of $\mathrm{D}$ - and T-loops, the anticodon stem, the connector between the acceptor and the D-stems, and the rarity of conserved nucleotides. Even residue U33 in the anticodon loop (marked by an *) is not conserved in all families, the noticeable exception being the methionine-specific tRNAs with C33. Numbering is according to (Sprinzl et al., 1998), R stands for purine, Y for pyrimidine, $T$ for thymine, and $\Psi$ for pseudouridine. The broken line recalls that in mammlian mt tRNA ${ }^{\operatorname{Ser}(A G Y)}$, the complete D-arm is missing. (B) Primary sequence conservation in mammalian $\mathrm{mt}$ tRNAs with the examples of tRNA $^{\text {Ilc }}$ (strongly conserved, left) and tRNA ${ }^{\text {Lys }}$ (partially conserved, right). The degree of conservation of each nucleotide within 31 mammalian sequences, are indicated by the background color $(100 \%$ conservation, dark; conservation over $90 \%$, light gray; conservation to more than $50 \%$ but less than $90 \%$, white). Squares indicate conservation of purines of pyrimidines, and circles indication non-conservation. Arrowheads in tRNA ${ }^{\text {Lys }}$ indicate the presence of additional nucleotides in some of the analyzed sequences. Data are according to Helm et al., 2000. 


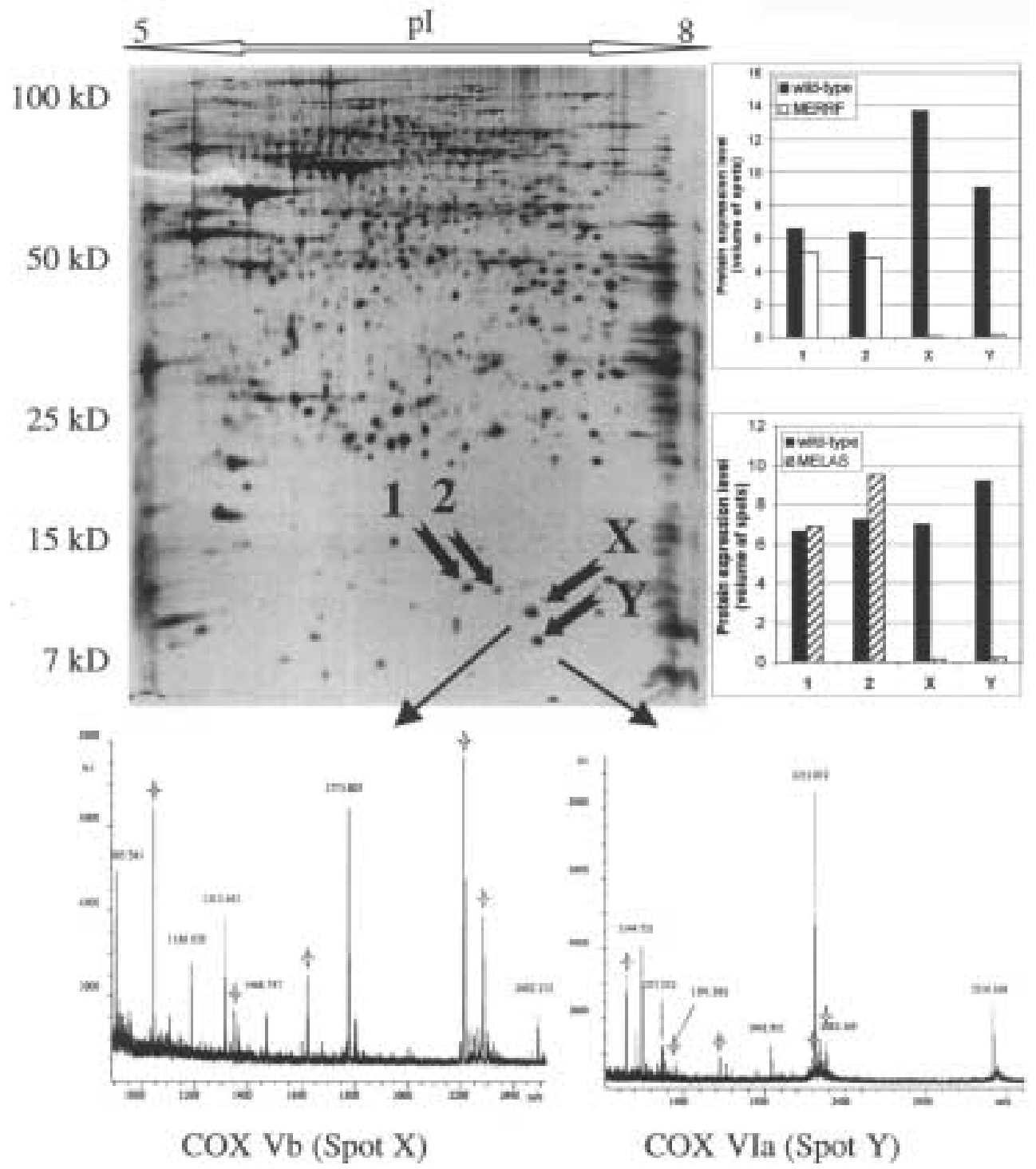

Fig. 3. Proteomics as a potential tool for the exploration of $\mathrm{mt}$ disorders. The approach includes twodimensional separation of human $\mathrm{mt}$ proteins by isoelectrofocusing and polyacrylamide gel electrophoresis. A typical silver-stained gel obtained on mitochondria extracted from a wild-type cybrid cell line is shown. Quantitative comparison between the 2D patterns obtained for wild-type and mutation-carrying mitochondria allows the determination of effects on proteins in the disease-representative mitochondria. Histograms in the right part of the figure illustrate this step by comparison of 4 spots in experiments performed to investigate the MERRF mutation A8344G in tRNA $^{\text {Lys }}$ (upper part) and the MELAS mutation A3243G in tRNA ${ }^{\text {Leu(UUR) }}$ (lower histogram). Quantification is done by PD-Quest software (BIO$\mathrm{RAD})$. In both couples, proteins in spots 1 and 2 are rather unaffected whereas proteins in spots $\mathrm{X}$ and $\mathrm{Y}$ are very strongly decreased in the disease-carrying mitochondria. Assignment of these two spots by mass spectrometric techniques (MALDI-TOF) revealed nuclear encoded subunits of cytochrome c oxidase, a respiratory chain complex (bottom). The approach is based on trypsin digestion of the proteins, and mass measurements of the different peptides (those peaks labeled with a star correspond to internal mass controls). The combination of masses is submitted to database searches and the best fits with protein sequences are reported. 
necessarily limited the number of proteins analyzed. Thanks to the sensitivity of mass spectrometric techniques, proteomics allows any protein seen on the gel to be assigned as long as its sequence is present in databases. Additionally, use of cybrid cell lines allows one to circumscribe the effects of a single point mutation in a given mt tRNA gene, rather than to determine effects of complete translation inhibition. Comparative proteomic analysis of $\mathrm{mt}$ proteins from various cybrid cell lines, representative of as many tRNA mutations as possible, or from biopsies, will certainly highlight sensitive distinctions in the patterns of affected proteins and thus contribute to our understanding on the relationships between genotypes and phenotypes. Proteomics has already proven to be very efficient in the understanding of diseases or in diagnosis (e.g., Celis et al., 2000). Further applications include mt disorders linked either to $\mathrm{mt}$ genome mutations in rRNA or protein genes, or to nuclear mutations (Schon, 2000). A recent investigation on $\mathrm{mt}$ proteins from neuroblastoma cell-lines representative of Alzheimer's disease illustrates these possibilities (Scheffler et al., 2001).

\section{CONCLUSION}

More than 115 different disease-related mutations have been found throughout the mt genome (Kogelnik et al., 1998) with 38\% occurring in protein genes (respiratory chain subunits), and $62 \%$ in $\mathrm{mt}$ protein synthesis related genes ( $4 \%$ in ribosomal and 58\% in tRNA genes). Since each tRNAs and rRNAs is involved in a common pathway, it could have been expected that all mutations taking place in the corresponding genes would lead to (i) a same molecular effect, namely a decreased rate of synthesis of the $13 \mathrm{mt}$-encoded proteins, (ii) a same metabolic consequence, namely decrease in ATP synthesis, and (iii) same phenotypic consequences. This is, however, a too simplisitc view, biased at many steps, so that today no common simple mechanistic process can explain the effects of the mutations. As briefly summarized in this review, the various combinations of model systems and mutations analyzed so far recall, indeed, that the translation process itself can be affected by tRNA mutations in many different ways, both qualitatively and quantitatively. These variations may well be dependent on the molecular environment of the affected tRNA within the mitochondria, environment which is likely different according to the nuclear background. Long-range effects of the mutations, as observed in initial proteomic studies, contribute to the expression of the disease as well. Further investigations both in the frame of $\mathrm{mt}$ protein biosynthesis and in the larger frame of integrated biological aspects of mitochondria in the cell, are clearly needed to better understand the genotype/phenotype relationships of mt tRNA disorders.

\section{ACKNOWLEDGMENTS}

I would like to thank M. Sissler and P. Tryoen-Toth for help in preparation of Tables and Figures, and C. Paulus, J. Pütz, M. Sissler, B. Sohm, P. Tryoen-Toth and R. Giegé for their contribution to the work on mt tRNAs. I am grateful to T. Rabilloud, J. Lunardi (CEA, Grenoble), A. Van Dorsselaer (LSMBO, Strasbourg), and members of their groups for the fruitful initial steps of a collaboration 
on mt proteomics. Special thanks go to L. Levinger for critical reading of the manuscript and extensive improvement of the English syntax. This work was supported by Centre National de la Recherche Scientifique (CNRS) and especially the Programme "Physique Chimie de Vivant," Université Louis Pasteur Strasbourg, Association Française contre les Myopathies (AFM), and European Community Grant QLG2CT-1999-00660.

\section{REFERENCES}

Allen, J. F. and Raven, J. A. (1996) Free-radical-induced mutation vs. redox regulation: costs and benefits of genes in organelles. J. Mol. Evol. 42:482-492.

Anderson, S. et al. (1981). Sequence and organization of the human mitochondrial genome. Nature 290:457-465.

Antonicka, H. et al. (1999) Defective kinetics of cytochrome c oxidase and alteration of mitochondrial membrane potential in fibroblasts and cytoplasmic hybrid cells with the mutation for myoclonus epilepsy with ragged-red fibres ('MERRF') at position 8344 nt. Biochem. J. 342:537-544.

Asoh, S., Mori, T., Hayashi, J.-I., and Ohta, S. (1996) Expression of the apoptosis-mediator Fas is enhanced by dysfunctional mitochondria. J. Biol. Chem. 120:600-607.

Bindoff, L. A. et al. (1993) Abnormal RNA processing associated with a novel tRNA mutation in mitochondrial DNA. A potential disease mechanism. J. Biol. Chem. 268:19559-19564.

Börner, G. V. et al. (2000) Decreased aminoacylation of mutant tRNAs in MELAS but not in MERRF patients. Hum. Mol. Genet. 9(4), 467-475.

Bullard, J., Cai, Y.-C., Demeler, B., and Spremulli, L. (1999) Expression and characterization of a human mitochondrial phenylalanyl-tRNA synthetase. J. Mol. Biol. 288:567-577.

Celis, J. et al. (2000) Gene expression profiling: monitoring transcription and translation products using DNA microarrays and proteomics. FEBS Letters 480:2-16.

Chomyn, A., Enriquez, J. A., Micol, V., Fernandez-Silva, P., and Attardi, G. (2000) The mitochondrial myopathy, encephalopathy, lactic acidosis, and stroke-like episode syndrome-associated human mitochondrial tRNA ${ }^{\text {Leu }}(\mathrm{UUR})$ mutation causes aminoacylation deficieny and concomitant reduced associated of mRNA with ribosomes. J. Biol. Chem. 275:19198-19209.

de Bruijn, M. H. L. and Klug, A. (1983) A model for the tertiary structure of mammalian mitochondrial transfer RNAs lacking the entire 'dihydrouridine' loop and stem. EMBO J. 2:1309-1321.

Degoul, F. et al. (1998) Isoleucylation properties of native human mitochondrial tRNA ${ }^{\text {Ile }}$ and tRNA ${ }^{\text {Ile }}$ transcripts. Implications for cardiomyopathy-related point mutations $(4269,4317)$ in the tRNA ${ }^{\text {Ile }}$ gene. Hum. Mol. Gen. 7(3), 347-354.

DiMauro, S. and Andreu, A. (2000). Mutations in mtDNA: are we scraping the bottom of the barrel? Brain Pathology 10:431-441.

DiMauro, S. and Moraes, C. T. (1993) Mitochondrial encephalomyopathies. Arch. Neurol. 50:1197-1208.

Dirheimer, G., Keith, G., Dumas, P., and Westhof, E. (1995) Primary, secondary and tertiary structures of tRNAs. In: tRNA: Structure, Biosynthesis, and Function. (Söll, D. and RajBhandary, U. L., eds.), Am. Soc. Microbiol. Press, Washington, DC. pp. 93-126.

Enriquez, J. A., Chomyn, A., and Attardi, G. (1995) MtDNA mutation in MERRF syndrome causes defective aminoacylation of $\mathrm{tRNA}^{\mathrm{Lys}}$ and premature translation termination. Nature Genetics 10:4755.

Flierl, A., Reichmann, H., and Seibel, P. (1997) Pathophysiology of the MELAS 3243 transition mutation. J. Biol. Chem. 272(43):27189-27196.

Florentz, C. and Sissler, M. (2001) Disease-related versus polymorphic mutations in human mitochondrial tRNas: where is the difference? EMBO Reports 2(6):481-486.

Giegé, R., Puglisi, J. D., and Florentz, C. (1993) tRNA structure and aminoacylation efficiency. Prog. Nucleic Acid Res. Mol. Biol. 45:129-206.

Giegé, R., Sissler, M., and Florentz, C. (1998) Universal rules and idiosyncratic features in tRNA identity. Nucleic Acids Res. 26:5017-5035. 
Goto, Y., Nonaka, I., and Horai, S. (1990) A mutation in the tRNA ${ }^{\text {Leu }}(U U R)$ gene associated with the MELAS subgroup of mitochondrial encephalomyopathies. Nature 348:651-653.

Hao, H. and Moraes, C. T. (1996) Functional and moleclar mitochondrial abnormalities associated with a C - > T transition at position 3526 of the human mitochondrial genome. The effects of a pathogenic mitochondrial tRNA point mutation in organelle translation and RNA processing. J. Biol. Chem. 271(4):2347-2352.

Hao, H. and Moraes, C. T. (1997) A disease-associated G5703A mutation in human mitochondrial DNA causes a conformational change and a marked decrease in steady-state levels of mitochondrial tRNA $^{\text {Asn }}$. Mol. Cel. Biol. 17(12):6831-6837.

Hayashi, I., Kawai, G., and Watanabe, K. (1998) Higher-order structure and thermal instability of bovine mitochondrial tRNA ${ }^{\text {Ser }}$ UGA investigated by proton NMR spectroscopy. J. Mol. Biol. 284:57-69.

Hayashi, I., Yokogawa, T., Kawai, G., Ueda, T., Nishikawa, K., and Watanabe, K. (1997) Assignment of imino proton signals of G-C base pairs and magnesium ion binding: an NMR study of bovine mitochondrial tRNA ${ }_{\text {GCU }}^{\mathrm{Ser}}$ lacking the entire D arm. J. Biochem. 121:1115-1122.

Helm, M. et al. (1998) The presence of modified nucleotides is required for cloverleaf folding of a human mitochondrial tRNA. Nucleic Acids Res. 26:1636-1643.

Helm, M., Brulé, H., Friede, D., Giegé, R., Pütz, J., and Florentz, C. (2000) Search for characteristic structural features of mammalian mitochondrial tRNAs. RNA 6:1356-1379.

Helm, M., Florentz, C., Chomyn, A., and Attardi, G. (1999a) Search for differences in post-transcriptional modification patterns of mitochondrial DNA-encoded wild-type and mutant human tRNA ${ }^{\text {Lys }}$ and tRNA ${ }^{\text {Leu(UUR) }}$. Nucleic Acids Res. 27:756-763.

Helm, M., Giegé, R., and Florentz, C. (1999b) A Watson-Crick base-pair disrupting methyl group ( ${ }^{1}$ A9) is sufficient for cloverleaf folding of human mitochondrial tRNA ${ }^{\text {Lys }}$. Biochemistry 38:13338-13346.

Ingman, M., Kaessmann, H., Pääbo, S., and Gyllenstein, U. (2000) Mitochondrial genome variation and the origin of modern humans. Nature 408:708-713.

Jacobs, H. T., and Holt, I. J. (2000). The np 3243 MELAS mutation: damned if you aminoacylate, damned if you don't. Hum. Mol. Genet. 9(4):463-465.

Kelley, S., Steinberg, S., and Schimmel, P. (2000) Functional defects of pathogenic human mitochondrial tRNAs related to structural fragility. Nature Struc. Biol. 7:862-865.

Kelley, S. O., Steinberg, S. V., and Schimmel, P. (2001) Fragile T-stem in Disease-associated Human Mitochondrial tRNA Sensitizes Structure to Local and Distant Mutations. J. Biol. Chem. 276(14):10607-10611.

King, M. P., Koga, Y., Davidson, M., and Schon, E. A. (1992) Defects in mitochondrial protein synthesis and respiratory chain activity segregate with the tRNAleu(UUR) mutation associated with mitochondrial myopathy, encephalopathy, lactic acidosis and strokelike episodes. Mol. Cell. Biol. 12(2):480-490.

Koga, Y., Davidson, M., Schon, E. A., and King, M. P. (1995) Analysis of cybrids harboring MELAS mutations in the mitochondrial tRNA ${ }^{\text {Leu(UUR) }}$ gene. Muscle \& Nerve Suppl 3:S119-S123.

Kogelnik, A. M., Lott, M. T., Brown, M. D., Navathe, S. B., and Wallace, D. C. (1998) MITOMAP: a human mitochondrial genome database-1998 update. Nucleic Acids Res. 26:112-115.

Krings, M., Stone, A., Schmitz, R. W., Krainitzki, H., Stoneking, M., and Pääbo, S. (1997) Neandertal DNA sequences and the origin of modern humans. Cell 90(1):19-30.

Larsson, N.-G. and Clayton, D. A. (1995). Molecular genetic aspects of human mitochondrial disorders. Annu. Rev. Genetics 29:151-178.

Levinger, L., Jacobs, O., and James, M. (2001) In vitro 3' end endonucleolytic processing defect in a human mitochondrial tRNA ${ }^{\text {Ser(UCN) }}$ precursor with the U7445C substitution, which causes non-syndromic deafness. Nucleic Acids Res. 29:4334-4340.

Martin, N. C. (1995) Organellar tRNAs: biosynthesis and function. In: tRNA: Structure, Biosynthesis, and Function. (Söll, D. and RajBhandary, U. L., eds.), ASM Press, Washington D.C. pp. 127-140.

Masucci, J. P. Davidson, M., Koga, Y., Schon, E. A., and King, M. P. (1995) In vitro analysis of mutations causing myoclonus epilepsy with ragged-red fibers in the mitochondrial tRNA ${ }^{\text {Lys }}$ gene: two genotypes produce similar phenotypes. Mol. Cell. Biol. 15(5):2872-2881.

Masucci, J. P. and Schon, E. A. (1996) tRNA processing in human mitochondrial disorders. Mol. Biol. Rep. 22:187-193. 
Mirabella, M., Di Giovanni, S., Silvestri, G., Tonali, P., and Servidei, S. (2000) Apoptosis in mitochondrial encephalomyopathies with mitochondrial DNA mutations: a potential pathogenic mechanism. Brain 123:93-104.

Montoya, J., Ojala, D., and Attardi, G. (1981) Distinctive features of the $5^{\prime}$-terminal sequences of the human mitochondrial mRNAs. Nature 290:465-470.

Nijtmans, L., Spelbrink, J., Van Galen, M., Zwaan, M., Klement, P., and Van den Bogert, C. (1995) Expression and fate of the nuclearly encoded subunits of cytochrome-c oxidase in cultured human cells depleted of mitochondrial gene products. Biochem. Biophys. Acta 1265:117-126.

O'Farrell, P. (1995) High resolution of 2-dimension electrophoresis of proteins. J. Mol. Biol. 250:40074021.

Pesole, G., Gissi, C., De Chirico, A., and Saccone, C. (1999) Nucleotide substitution rate of mammalian mitochondrial genomes. J. Mol. Evol. 48:427-434.

Rabilloud, T. et al. (1998) Two-dimensional electrophoresis of human placental mitochondria and protein identification by mass spectrometry: toward a human mitochondrial proteome. Electrophoresis 19:1006-1014.

Rabilloud, T. et al. (2002) Comparative proteomics as a new tool for exploring human mitochondrial tRNA disorders. Biochemistry, 41:144-150.

Rossmanith, W. and Karwan, R. (1998) Impairment of tRNA processing by point mutations in mitochondrial tRNA ${ }^{\text {Leu(UUR) }}$ associated with mitochondrial diseases. FEBS Letters 433:269-274.

Scheffler, I. (1999) Mitochondria, John Wiley and Sons, Inc., Publication, New York, Chichester, Weinheim, Brisbane, Singapore, Toronto.

Scheffler, N. et al. (2001) Two-dimensional electrophoresis and mass spectrometric identification of mitochondrial proteins from as SH-SY5Y neuroblastoma cell line. Mitochondrion 1:161-179.

Schon, E. (2000) Mitochondrial genetics and disease. TIBS, 555-560.

Schon, E. A., Bonilla, E., and DiMauro, S. (1997) Mitochondrial DNA mutations and pathogenesis. $J$. Bioenerg. Biomemb. 29(2):131-149.

Schon, E. A., Koga, Y., Davidson, M., Moraes, C. T., and King, M. P. (1992) The mitochondrial tRNA $^{\text {Leu(UUR) }}$ mutation in MELAS: A model for pathogenesis. Biochim. Biophys. Acta 1101:206209.

Seibel, P. et al. (1991) Genetic biochemical and pathophysiological characterization of a familial mitochondrial encephalomyopathy (MERRF). J. Neurol. Sci. 105:217-224.

Söll, D. and RajBhandary, U. L., Eds. (1995) tRNA: Structure, Biosynthesis, and Function. Washington, DC: Am. Soc. Microbiol. Press.

Sprinzl, M., Horn, C., Brown, M., Ioudovitch, A., and Steinberg, S. (1998). Compilation of tRNA sequences and sequences of tRNA genes. Nucleic Acids Res. 26:148-153.

Tolkunova, E., Park, H., Xia, J., King, M. P., and Davidson, E. (2000) The human lysyl-tRNA synthetase gene encodes both the cytoplasmic and mitochondrial enzymes by means of an unusual splicing of the primary transcript. J. Biol. Chem. 275:35063-35069.

Ueda, T., Watanabe, K., and Ohta, T. (1983). Structural analysis of bovine mitochondrial tRNA ${ }^{\text {Ser(AGY) }}$. Nucleic Acids Res. Symposium Series 12:141-144.

Wakita, K. et al. (1994) Higher-order structure of bovine mitochondrial tRNAPhe lacking the 'conserved'

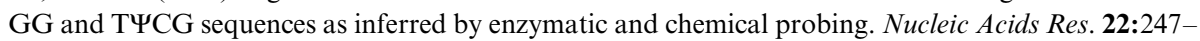
353.

Wallace, D. C. (1999). Mitochondrial diseases in man and mouse. Science 283:1482-1488.

Watanabe, Y. I. et al. (1994) Higher-order structure of bovine mitochondrial tRNA ${ }^{\text {Ser }}$ UGA: Chemical modification and computer modeling. Nucleic Acids Res. 22(24):5378-5384.

Wilkins, M., Williams, K., Appel, R., and Hochstrasser, D., Eds. (1997) Proteome research: new frontiers in functional genomics. Principles and Practice. Heidelberg: Springer Verlag.

Wolstenholme, D. R., Macfarlane, J. L., Okimoto, R. Clary, D. O., and Wahleithner, J. A. (1987) Bizarre tRNAs inferred from DNA sequences of mitochondrial genomes of nematode worms. Proc. Natl. Acad. Sci. U.S.A. 84:1324-1328.

Yasukawa, T., Suzuki, T., Ishii, N., Ohta, S., and Watanabe, K. (2001). Wobble modification defect in tRNA disturbs codon-anticodon interaction in a mitochondrial disease. EMBO Journal 20:47944802 . 
Yasukawa, T., Hino, N., Suzuki, T., Watanabe, K., Ueda, T., and Ohta, S. (2000a) A pathogenic point mutation reduces stability of mitochondrial mutant tRNA ${ }^{\text {Ile }}$. Nucleic Acids Res. 28:3779-3784.

Yasukawa, T., Suzuki, T., Ishii, N., Ueda, T., Ohta, S., and Watanabe, K. (2000b) Defect in modification at the anticodon wobble nucleotide of mitochondrial tRNA ${ }^{\text {Lys }}$ with the MERRF encephalomyopathy pathogenic mutation. Febs Lett. 467:175-178.

Yasukawa, T., Suzuki, T., Suzuki, T., Ueda, T., Ohta, S., and Watanabe, K. (2000c) Modification defect at anticodon wobble nucleotide of mitochondrial tRNA ${ }^{\mathrm{Leu}(\mathrm{UUR})}$ with pathogenic mutations of mitochondrial myopathy, encephalopathy, lactic acidosis and stroke-like episodes. J. Biol. Chem. 275:4251-4257. 